\title{
Sinogram Restoration for Low-Dosed X-Ray Computed Tomography Using Fractional-Order Perona-Malik Diffusion
}

\author{
Shaoxiang Hu, ${ }^{1}$ Zhiwu Liao, ${ }^{2}$ and Wufan Chen ${ }^{3}$ \\ ${ }^{1}$ School of Automation Engineering, University of Electronic Science and Technology of China, \\ Chengdu 611731, China \\ ${ }^{2}$ School of Computer Science, Sichuan Normal University, Chengdu 610101, China \\ ${ }^{3}$ Institute of Medical Information and Technology, School of Biomedical Engineering, \\ Southern Medical University, Guangzhou 510515, China \\ Correspondence should be addressed to Zhiwu Liao, liaozhiwu@163.com
}

Received 18 January 2012; Accepted 16 March 2012

Academic Editor: Ming Li

Copyright (c) 2012 Shaoxiang Hu et al. This is an open access article distributed under the Creative Commons Attribution License, which permits unrestricted use, distribution, and reproduction in any medium, provided the original work is properly cited.

Existing integer-order Nonlinear Anisotropic Diffusion (NAD) used in noise suppressing will produce undesirable staircase effect or speckle effect. In this paper, we propose a new scheme, named Fractal-order Perona-Malik Diffusion (FPMD), which replaces the integer-order derivative of the Perona-Malik (PM) Diffusion with the fractional-order derivative using G-L fractional derivative. FPMD, which is a interpolation between integer-order Nonlinear Anisotropic Diffusion (NAD) and fourth-order partial differential equations, provides a more flexible way to balance the noise reducing and anatomical details preserving. Smoothing results for phantoms and real sinograms show that FPMD with suitable parameters can suppress the staircase effects and speckle effects efficiently. In addition, FPMD also has a good performance in visual quality and root mean square errors (RMSE).

\section{Introduction}

Radiation exposure and associated risk of cancer for patients receiving CT examination have been an increasing concern in recent years. Thus minimizing the radiation exposure to patients has been one of the major efforts in modern clinical X-ray CT radiology [1-8].

A simple and cost-effective means to achieve low-dose CT applications is to lower Xray tube current $(\mathrm{mA})$ as low as achievable $[6,7]$. However, the presentation of strong noise degrades the quality of low-dose CT images dramatically and decreases the accuracy of the diagnosis dose.

Filtering noise from clinical scans is a challenging task, since these scans contain artifacts and consist of many structures with different shape, size, and contrast, which should be 
preserved for making correct diagnosis. Many strategies have been proposed to reduce the noise, for example, by nonlinear noise filters [8-20] and statistics-based iterative image reconstructions (SIIRs) [21-29].

The SIIRs utilize the statistical information of the measured data to obtain good denoising results but are limited for their excessive computational demands for the large CT image size. Although the nonlinear filters show effectiveness in reducing noise both in sinogram space and image space, they cannot handle the noise-induced streak artifacts. Some nonlinear filters, such as Nonlinear Anisotropic Diffusion (NAD), even produce new artifacts in L-CT denoising [30-37].

To eliminate the undesirable staircase effect, high-order PDEs (typically fourth-order PDEs) for image restoration have been introduced in [38-43]. Though these methods can eliminate the staircase effect efficiently, they often lead to a speckle effect [44].

Recently, fractional-order PDEs have been studied in many fields [38-49]. The fractional derivative can be seen as the generalization of the integer-order derivative. It has been studied by many mathematicians (e.g., Euler, Hardy, Littlewood, and Liouville) [47]. Not until Mandelbrot found fractals and applied the G-L fractional derivative to the Brownian motion did the fractional derivative cause great attention. There are many methods that can define the fractional derivative. The usual definitions among them involve G-L fractional derivative, Cauchy-integral fractional derivative, frequency-domain (Fourier-domain) fractional derivative.

$\mathrm{Li}$ and Zhao investigate relation between the data of cyber-physical networking systems and power laws and then suggest that power-law-type data may be governed by stochastically differential equations of fractional order [45]. They also propose that one-dimensional random functions with long-range dependence (LRD) based on a specific class of processes called the Cauchy-class (CC) process maybe a possible model of sea level data [46].

You and Kaveh develop a class of fractional-order multiscale variational model using G-L definition of fractional-order derivative and propose an efficient condition of the convergence for the model [38]. The experiments show that the model can improve the peak signalto-noise ratio, preserve texture, and eliminate the stair effect efficiently.

Bai and Feng proposed a class of fractional-order anisotropic diffusion equations based on PM equation for image denoising using Fourier-domain fractional derivative in [49]. The numerical results showed that both of the staircase effect and the speckle effect can be eliminated effectively by using the fractional-order derivative.

Inspired from previous works and in order to eliminate the staircase effects and preserve anatomical details, we propose to replace the first-order and the second-order derivative of the PM Diffusion with the fractional-order derivative using G-L fractional derivative. It should be indicated that the method proposed in this paper, which is carried on the sinogram space directly, is different to the method proposed in [49], which is carried on the Fourier space.

The arrangement of this paper is as follows. In Section 2, the noise model of Low-dosed CT (L-CT) is introduced; and then the PM diffusion is given in Section 3, new fractional-order PM method is developed using G-L fractional definition in Section 4; the experiment results are shown and discussed in Section 5; the final part is the conclusions and acknowledgement.

\section{Noise Models}

Based on repeated phantom experiments, low-mA (or low-dose) CT-calibrated projection data after logarithm transform were found to follow approximately a Gaussian distribution 
with an analytical formula between the sample mean and sample variance, that is, the noise is a signal-dependent Gaussian distribution [20].

In this section, we will introduce signal-independent Gaussian noise (SIGN), Poisson noise, and signal-dependent Gaussian noise.

\subsection{Signal-Independent Gaussian Noise (SIGN)}

SIGN is a common noise for imaging system. Let the original projection data be $\left\{x_{i}\right\}, i=1$, $\ldots, m$, where $i$ is the index of the $i$ th bin. The signal has been corrupted by additive noise $\left\{n_{i}\right\}, i=1, \ldots, m$ and one noisy observation

$$
y_{i}=x_{i}+n_{i}
$$

where $y_{i}, x_{i}, n_{i}$ are observations for the random variables $Y_{i}, X_{i}$, and $N_{i}$ where the uppercase letters denote the random variables and the lower-case letters denote the observations for respective variables. $X_{i}$ is normal $N\left(0, \sigma_{X}^{2}\right) ; N_{i}$ is normal $N\left(0, \sigma_{N}^{2}\right)$ and independent to the Gaussian random variable $X_{i}$. Thus $Y_{i}$ is normal $N\left(0, \sigma_{X}^{2}+\sigma_{N}^{2}\right)$.

\subsection{Poisson Model and Signal-Dependent Gaussian Model}

The photon noise is due to the limited number of photons collected by the detector [36]. For a given attenuating path in the imaged subject, $N_{0}(i, \alpha)$ and $N(i, \alpha)$ denote the incident and the penetrated photon numbers, respectively. Here, $i$ denote the index of detector channel or bin, and $\alpha$ is the index of projection angle. In the presence of noises, the sinogram should be considered as a random process and the attenuating path is given by

$$
r_{i}=-\ln \left[\frac{N(i, \alpha)}{N_{0}(i, \alpha)}\right]
$$

where $N_{0}(i, \alpha)$ is a constant and $N(i, \alpha)$ is Poisson distribution with mean $N$.

Thus we have

$$
N(i, \alpha)=N_{0}(i, \alpha) \exp \left(-r_{i}\right)
$$

Both its mean value and variance are $N$.

Gaussian distributions of ploy-energetic systems were assumed based on limited theorem for high-flux levels and followed many repeated experiments in [20]. We have

$$
\sigma_{i}^{2}\left(\mu_{i}\right)=f_{i} \exp \left(\frac{\mu_{i}}{\gamma}\right)
$$

where $\mu_{i}$ is the mean and $\sigma_{i}^{2}$ is the variance of the projection data at detector channel or bin $i$, $\gamma$ is a scaling parameter, and $f_{i}$ is a parameter adaptive to different detector bins.

The most common conclusion for the relation between Poisson distribution and Gaussian distribution is that the photon count will obey Gaussian distribution for the case 
with large incident intensity and Poisson distribution with feeble intensity [20]. In addition, in [36], the authors deduce the equivalency between Poisson model and Gaussian model. Therefore, both theories indicate that these two noises have similar statistical properties and can be unified into a whole framework.

\section{Perona-Malik Diffusion}

In image smoothing, Nonlinear Anisotropic Diffusion (NAD), also called Perona-Malik diffusion (PMD), is a technique aiming at reducing image details without removing significant parts of the image contents, typically edges, lines, or textures, which are important for the image [50].

With a constant diffusion coefficient, the anisotropic diffusion equations reduce to the heat equation, which is equivalent to Gaussian blurring. This is ideal for smoothing details but also blurs edges. When the diffusion coefficient is chosen as an edge seeking function, the resulting equations encourage diffusion (hence smoothing) within regions and stop it near strong edges. Hence the edges can be preserved while smoothing from the image [50].

Formally, NAD is defined as

$$
\frac{\partial u(x, y, t)}{\partial t}=\operatorname{div}(g(x, y, t) \nabla u(x, y, t))
$$

where $u(x, y, 0)$ is the initial gray scale image, $u(x, y, t)$ is the smooth gray scale image at time $t, \nabla$ denotes the gradient, $\operatorname{div}(\cdot)$ is the divergence operator, and $g(x, y, t)$ is the diffusion coefficient. $g(x, y, t)$ controls the rate of diffusion and is usually chosen as a monotonically decreasing function of the module of the image gradient. Two functions proposed in [50] are

$$
\begin{gathered}
g(\|\nabla u(x, y, t)\|)=e^{-(\|\nabla u(x, y, t)\| / \sigma)^{2}}, \\
g(\|\nabla u(x, y, t)\|)=\frac{1}{1+(\|\nabla u(x, y, t)\| / \sigma)^{2}},
\end{gathered}
$$

where $\|\cdot\|$ is the module of the vector and the constant $\sigma$ controls the sensitivity to edges.

Perona and Malik propose a simple method to approach the modules of gradients, which is called PM diffusion [50]. Its discretization for Laplacian operator is

$$
\begin{aligned}
u(i, j, t+1)=u(i, j, t)+\frac{1}{4}[ & \quad c_{N} \cdot \nabla_{N}^{2} u(i, j, t) \\
& \left.+c_{S} \cdot \nabla_{S}^{2} u(i, j, t)+c_{E} \cdot \nabla_{E}^{2} u(i, j, t)+c_{W} \cdot \nabla_{W}^{2} u(i, j, t)\right],
\end{aligned}
$$

where

$$
\begin{aligned}
& \nabla_{N}^{2} u(i, j, t)=u(i-1, j, t)-u(i, j, t), \\
& \nabla_{S}^{2} u(i, j, t)=u(i+1, j, t)-u(i, j, t),
\end{aligned}
$$




$$
\begin{aligned}
\nabla_{E}^{2} u(i, j, t) & =u(i, j+1, t)-u(i, j, t), \\
\nabla_{W}^{2} u(i, j, t) & =u(i, j-1, t)-u(i, j, t) .
\end{aligned}
$$

According to (3.2)-(3.3), the diffusion coefficient is defined as a function of module of the gradient. However, computing a gradient accurately in discrete data is very complex and the module of the gradient is simplified as the absolute values of four directions and diffusion coefficients are

$$
\begin{aligned}
& c_{N}(i, j, t)=g\left(\left|\nabla_{N}^{2} u(i, j, t)\right|\right), \\
& c_{S}(i, j, t)=g\left(\left|\nabla_{S}^{2} u(i, j, t)\right|\right), \\
& c_{E}(i, j, t)=g\left(\left|\nabla_{E}^{2} u(i, j, t)\right|\right), \\
& c_{W}(i, j, t)=g\left(\left|\nabla_{W}^{2} u(i, j, t)\right|\right),
\end{aligned}
$$

where $|\cdot|$ is the absolute value of the number and $g(\cdot)$ is defined in (3.2) or (3.3).

The main default for PM diffusion is that it will lead to staircase effect or sometimes details oversmoothing. In order to eliminate the staircase effects and preserve anatomical details, we propose to replace the first-order and the second-order derivative of the PM Diffusion with the fractional-order derivative using G-L fractional derivative. The new diffusion model will be introduced in the next section.

\section{The Fractional-Order PM Diffusion (FPMD)}

The FPMD is developed using G-L fractional-order derivative, which is defined as [38]

$$
D^{\alpha} g(x)=\lim _{h \rightarrow 0^{+}} \frac{\sum_{k \geq 0}(-1)^{k} C_{k}^{\alpha} g(x-k h)}{h^{\alpha}}, \quad \alpha>0,
$$

where $g(x)$ is a real function, $\alpha>0$ is a real number, $C_{k}^{\alpha}=\Gamma(\alpha+1) /[\Gamma(k+1) \Gamma(\alpha-k+1)]$ is the generalized binomial coefficient and $\Gamma(\cdot)$ denotes the Gamma function. If $h=1$, the finite fractional difference is

$$
\Delta^{\alpha} g(x)=\sum_{k=0}^{K-1}(-1)^{k} C_{k}^{\alpha} g(x-k)
$$

An image $U$ will be a 2-dimensional matrix of size $N \times N$ and its discrete fractionalorder gradient $\nabla^{\alpha} u$ is an 8-dimensional vector:

$$
\begin{aligned}
& \nabla^{\alpha} \mathbf{u}(\mathbf{i}, \mathbf{j}) \\
& \quad=\left(\nabla_{0}^{\alpha} u(i, j), \nabla_{1}^{\alpha} u(i, j), \nabla_{2}^{\alpha} u(i, j), \nabla_{3}^{\alpha} u(i, j), \nabla_{4}^{\alpha} u(i, j), \nabla_{5}^{\alpha} u(i, j), \nabla_{6}^{\alpha} u(i, j), \nabla_{7}^{\alpha} u(i, j)\right)^{T},
\end{aligned}
$$

where $T$ represents the transpose of the vector and $\nabla^{\alpha} u_{k}(i, j), k=0, \ldots, 7$ are defined as

$$
\nabla_{0}^{\alpha} u(i, j)=\sum_{k=0}^{K-1}(-1)^{k} C_{k}^{\alpha} u(i, j+k), \quad \nabla_{1}^{\alpha} u(i, j)=\sum_{k=0}^{K-1}(-1)^{k} C_{k}^{\alpha} u(i-k, j+k),
$$




$$
\begin{array}{ll}
\nabla_{2}^{\alpha} u(i, j)=\sum_{k=0}^{K-1}(-1)^{k} C_{k}^{\alpha} u(i-k, j), & \nabla_{3}^{\alpha} u(i, j)=\sum_{k=0}^{K-1}(-1)^{k} C_{k}^{\alpha} u(i-k, j-k), \\
\nabla_{4}^{\alpha} u(i, j)=\sum_{k=0}^{K-1}(-1)^{k} C_{k}^{\alpha} u(i, j-k), & \nabla_{5}^{\alpha} u(i, j)=\sum_{k=0}^{K-1}(-1)^{k} C_{k}^{\alpha} u(i+k, j-k), \\
\nabla_{6}^{\alpha} u(i, j)=\sum_{k=0}^{K-1}(-1)^{k} C_{k}^{\alpha} u(i+k, j), & \nabla_{7}^{\alpha} u(i, j)=\sum_{k=0}^{K-1}(-1)^{k} C_{k}^{\alpha} u(i+k, j+k) .
\end{array}
$$

Thus

$$
\begin{aligned}
& \nabla^{2 \alpha} \mathbf{u}(\mathbf{i}, \mathbf{j}) \\
& =\left(\nabla_{0}^{2 \alpha} u(i, j), \nabla_{1}^{2 \alpha} u(i, j), \nabla_{2}^{2 \alpha} u(i, j), \nabla_{3}^{2 \alpha} u(i, j), \nabla_{4}^{2 \alpha} u(i, j), \nabla_{5}^{2 \alpha} u(i, j), \nabla_{6}^{2 \alpha} u(i, j), \nabla_{7}^{2 \alpha} u(i, j)\right)^{T},
\end{aligned}
$$

where $T$ represents the transpose of the vector. From (4.3), we have

$$
\begin{array}{ll}
\nabla_{0}^{2 \alpha} u(i, j)=\sum_{k=0}^{K-1}(-1)^{k} C_{k}^{\alpha} \nabla_{0}^{\alpha} u(i, j+k), & \nabla_{1}^{2 \alpha} u(i, j)=\sum_{k=0}^{K-1}(-1)^{k} C_{k}^{\alpha} \nabla_{1}^{\alpha} u(i-k, j+k), \\
\nabla_{2}^{2 \alpha} u(i, j)=\sum_{k=0}^{K-1}(-1)^{k} C_{k}^{\alpha} \nabla_{2}^{\alpha} u(i-k, j), & \nabla_{3}^{2 \alpha} u(i, j)=\sum_{k=0}^{K-1}(-1)^{k} C_{k}^{\alpha} \nabla_{3}^{\alpha} u(i-k, j-k), \\
\nabla_{4}^{2 \alpha} u(i, j)=\sum_{k=0}^{K-1}(-1)^{k} C_{k}^{\alpha} \nabla_{4}^{\alpha} u(i, j-k), & \nabla_{5}^{2 \alpha} u(i, j)=\sum_{k=0}^{K-1}(-1)^{k} C_{k}^{\alpha} \nabla_{5}^{\alpha} u(i+k, j-k), \\
\nabla_{6}^{2 \alpha} u(i, j)=\sum_{k=0}^{K-1}(-1)^{k} C_{k}^{\alpha} \nabla_{6}^{\alpha} u(i+k, j), & \nabla_{7}^{2 \alpha} u(i, j)=\sum_{k=0}^{K-1}(-1)^{k} C_{k}^{\alpha} \nabla_{7}^{\alpha} u(i+k, j+k) .
\end{array}
$$

Let

$$
\mathbf{g}=\left(g_{0}, g_{1}, g_{2}, g_{3}, g_{4}, g_{5}, g_{6}, g_{7}\right)^{T}
$$

where $T$ represents the transpose of the vector and $g_{k}, k=0, \ldots, 7$ is defined as

$$
g_{k}=\frac{g\left(\left|\nabla_{k}^{\alpha} u(i, j)\right|\right)}{\sum_{n=0}^{7} g\left(\left|\nabla_{n}^{\alpha} u(i, j)\right|\right)}, \quad k=0,1, \ldots, 7
$$

where $\nabla_{k}^{\alpha} u(i, j), k=0, \ldots, 7$, defined in (4.3) are the components of vector $\nabla^{\alpha} \mathbf{u}(\mathbf{i}, \mathbf{j})$ and $\sum_{n=0}^{7} g\left(\left|\nabla_{n}^{\alpha} u(i, j)\right|\right)$ is the normalized constant, $g$ is the decreasing function of absolute value 
of $\nabla_{k}^{\alpha} u(i, j), k=0, \ldots, 7$. Following (2.2) and (2.3), $g\left(\left|\nabla u_{k}^{\alpha}(x, y, t)\right|\right)$ can be defined as

$$
g\left(\left|\nabla u_{k}^{\alpha}(x, y, t)\right|\right)=e^{-\left(\left|\nabla u_{k}^{\alpha}(x, y, t)\right| / \sigma\right)^{2}}, \quad k=0, \ldots, 7
$$

or

$$
g\left(\left|\nabla u_{k}^{\alpha}(x, y, t)\right|\right)=\frac{1}{1+\left(\left|\nabla u_{k}^{\alpha}(x, y, t)\right| / \sigma\right)^{2}}, \quad k=0, \ldots, 7
$$

where $|\cdot|$ is the absolute value of the number and the constant $\sigma$ controls the sensitivity to edges.

The new FPMD based on G-L fractional-order derivative is defined as

$$
\frac{\partial u(i, j, t)}{\partial t}=\operatorname{div}\left(\begin{array}{l}
g_{0} \nabla_{0}^{\alpha} u(i, j, t) \\
g_{1} \nabla_{1}^{\alpha} u(i, j, t) \\
g_{2} \nabla_{2}^{\alpha} u(i, j, t) \\
g_{3} \nabla_{3}^{\alpha} u(i, j, t) \\
g_{4} \nabla_{4}^{\alpha} u(i, j, t) \\
g_{5} \nabla_{5}^{\alpha} u(i, j, t) \\
g_{6} \nabla_{6}^{\alpha} u(i, j, t) \\
g_{7} \nabla_{7}^{\alpha} u(i, j, t)
\end{array}\right),
$$

where the $\nabla_{k}^{\alpha} u(i, j, t), k=0, \ldots, 7$, are the components of vector $\nabla^{\alpha} \mathbf{u}(\mathbf{i}, \mathbf{j}, \mathbf{t})$ in (4.3) and $g_{k}$, $k=0, \ldots, 7$, defined in (4.8) are the components of $\mathbf{g}$ in (4.7).

The above equation can be represented as

$$
\frac{\partial u(i, j, t)}{\partial t}=\sum_{k=0}^{7} g_{k} \nabla_{k}^{2 \alpha} u(i, j, t),
$$

where $\sum_{k=0}^{7} g_{k}=1$ and $\nabla_{k}^{2 \alpha} u(i, j, t)$ can be computed according to (4.5).

Thus the explicit form for solving (4.12) is

$$
u(i, j, t+1)=u(i, j, t)+\sum_{k=0}^{7} g_{k} \nabla_{k}^{2 \alpha} u(i, j, t)
$$

where $u(i, j, t+1)$ is the gray level of $(i, j)$ at time $t+1$ and $g_{k}, \nabla_{k}^{2 \alpha} u(i, j, t)$ are the same as in (4.12).

\section{Experiments and Discussion}

The main objective for smoothing L-CT images is to delete the noise while to preserve anatomy details for the images. 
Table 1: RMSE of different smoothing methods.

\begin{tabular}{llccccccc}
\hline & Noisy & Median & Wlener & Gaussian & \multirow{2}{*}{ PMD } & FPMD & FPMD & FPMD \\
& image & Filter & Filter & Filter & & $\alpha=0.2$ & $\alpha=0.5$ & $\alpha=1.5$ \\
\hline RMSE & 0.0962 & 0.0804 & 0.0634 & 0.0963 & 0.0774 & 0.0603 & 0.0735 & 0.0752 \\
\hline
\end{tabular}

In order to show the performance of FPMD, a 2-dimensional $256 \times 256$ Shepp-Logan head phantom developed in MatLab. The number of bins per view is 888 with 984 views evenly spanned on a circular orbit of $360^{\circ}$. The detector arrays are on an arc concentric to the X-ray source with a distance of $949.075 \mathrm{~mm}$. The distance from the rotation center to the $X$-ray source is $541 \mathrm{~mm}$. The detector cell spacing is $1.0239 \mathrm{~mm}$. The L-CT projection data (sinogram) is simulated by adding Gaussian-dependent noise (GDN) whose analytic form between its mean and variance has been shown in (2.4). In this paper, set $f_{i}=4.0$ and $T=$ $2 e+4$. The projection data is reconstructed by standard Filtered Back Projection (FBP). Since both the original projection data and sinogram have been provided, the evaluation based on root-mean-square error (RMSE) between the ideal reconstructed image is and reconstructed images defined as

$$
\sqrt{\frac{1}{256 \times 256} \sum_{i=1}^{256} \sum_{j=1}^{256}\left(f_{\text {recon }}(i, j)-f_{\mathrm{Ph}}(i, j)\right)^{2}},
$$

where $f_{\text {recon }}(i, j)$ denotes the reconstructed value on position $(i, j)$ while $f_{\mathrm{Ph}}(i, j)$ denotes the ideal reconstructed value on position $(i, j)$.

Two abdominal CT images of a 62-year-old woman with different doses were scanned from a 16 multidetector row CT unit (Somatom Sensation 16; Siemens Medical Solutions) using $120 \mathrm{kVp}$ and $5 \mathrm{~mm}$ slice thickness. Other remaining scanning parameters are gantry rotation time, 0.5 second; detector configuration (number of detector rows section thickness), $16 \times 1.5 \mathrm{~mm}$; table feed per gantry rotation, $24 \mathrm{~mm}$; pitch, $1: 1$ and reconstruction method, Filtered Back Projection (FBP) algorithm with the soft-tissue convolution kernel "B30f". Different CT doses were controlled by using two different fixed tube current $30 \mathrm{mAs}$ and $150 \mathrm{mAs}$ ((60 mA or $300 \mathrm{mAs})$ for L-CT and standard-dose CT (SDCT) protocols, resp.). The CT dose index volume (CTDIvol) for LDCT images and SDCT images are in positive linear correlation to the tube current and are calculated to be approximately ranged between 15.32 mGy to 3.16 mGy [51] (see Figures 2(a) and 2(b)).

On sinogram space, FPMD with $\alpha=0.2, \alpha=0.5$, and $\alpha=1.5$ is carried on two image collections. Other compared methods include median filter with $5 \times 5$ window; wiener filter with $5 \times 5$ window; Gaussian filter whose mean is 0 and its standard deviation is 1.8. The diffusion coefficient for PMD and FPMDs is selected as a Gaussian function whose standard deviation is 2. All smoothed projection data will be reconstructed by standard FBP.

Table 1 summarized RMSE between the ideal reconstructed image and filtered reconstructed image. The FPMD with $\alpha=0.2$ has the best performance in RMSE, while other FPMD with $\alpha=0.5$ and $\alpha=1.5$ also has better performance than almost other comparing methods except for $5 \times 5$ wiener. In summary, the FPMD has a very good performance in RMSE. Since FPMD provides a more flexible way for diffusion than PMD, FPMD has much good performance in denoising while preserving structures. 


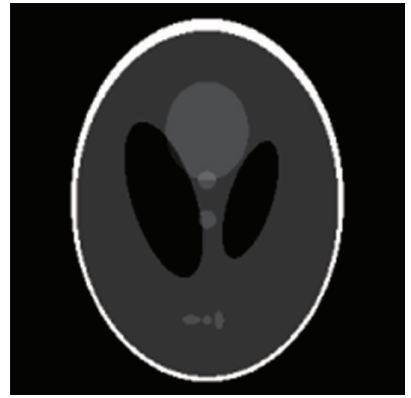

(a)

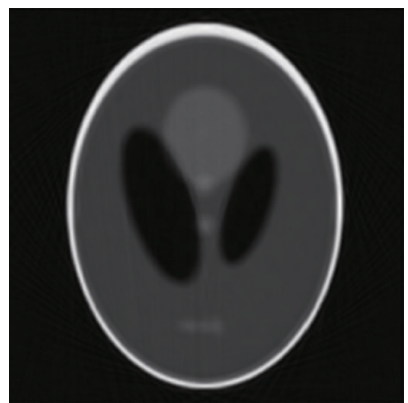

(d)

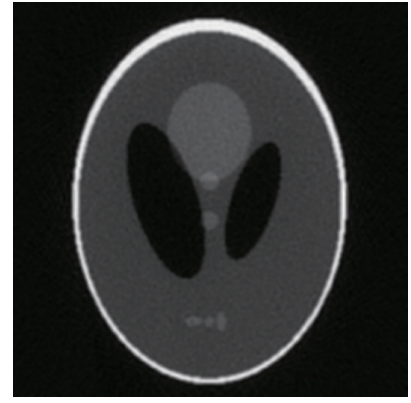

(g)

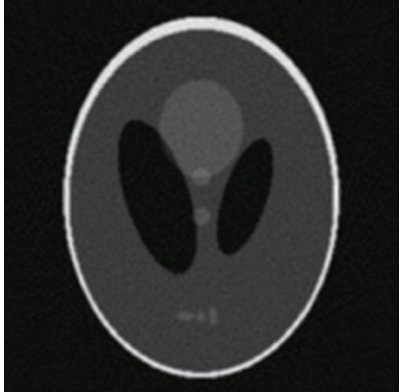

(b)

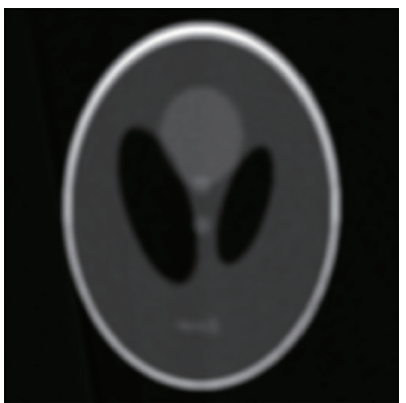

(e)

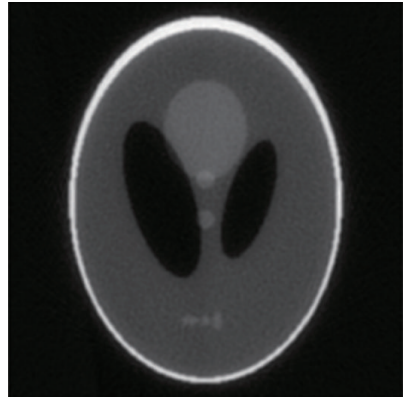

(h)

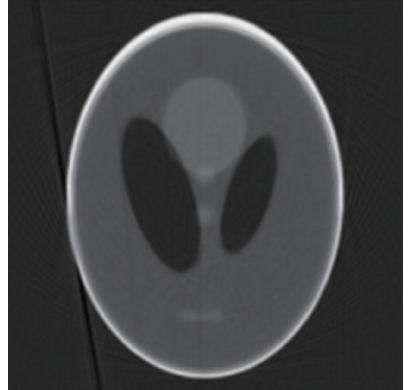

(c)

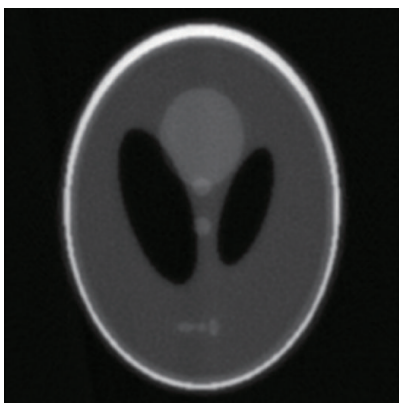

(f)

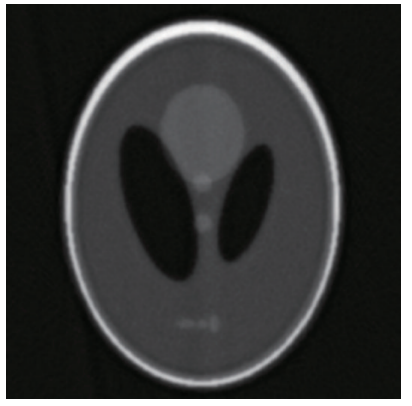

(i)

Figure 1: Shepp-Logan phantoms. (a) Original ideal reconstructed phantom. (b) Simulated LDCT reconstructed phantom. (c) LDCT reconstructed phantom processed by $5 \times 5$ median filter. (d) LDCT reconstructed phantom processed by $5 \times 5$ wiener filter. (e) LDCT reconstructed phantom processed by Gaussian smoothing with $[\sigma=1.8, \mu=0]$. (f) LDCT reconstructed phantom processed by PMD with $[\sigma=2]$. (g) LDCT reconstructed phantom processed by FPMD with $[\sigma=2, \alpha=0.2]$. (h) LDCT reconstructed phantom processed by FPMD with $[\sigma=2, \alpha=0.5]$. (i) LDCT reconstructed phantom processed by FPMD with $[\sigma=2, \alpha=1.5]$.

Comparing all the original SDCT images and L-CT images in Figures 1 and 2, we found that the L-CT images were severely degraded by nonstationary noise and streak artifacts. In Figures 2(g)-2(i), for the proposed FPMD approach, experiments with fractional-order $\alpha$ gradually increased will obtain more smooth images. Both in Figure 1 and 2, we can observe better noise/artifacts suppression and edge preservation when $\alpha=0.2$. Especially, compared to their corresponding original SDCT images, the fine features representing the intrahepatic bile duct dilatation and the hepatic cyst were well restored by using the 


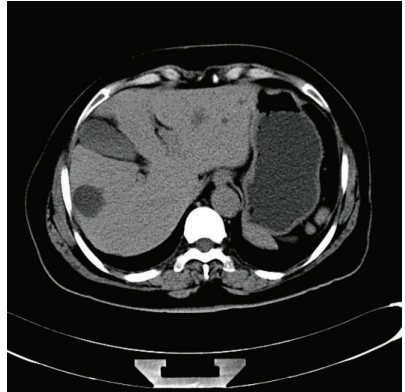

(a)

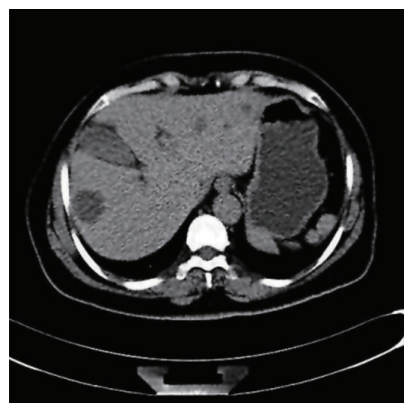

(d)

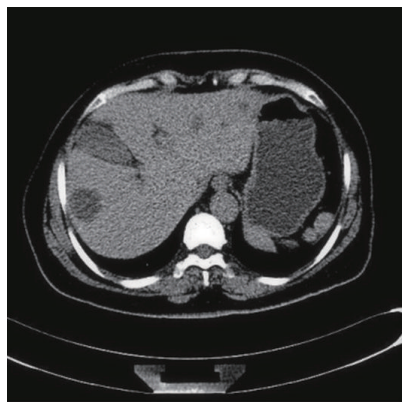

(g)

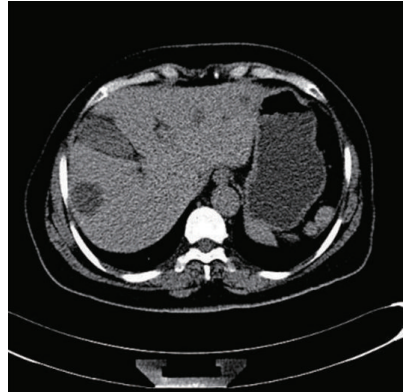

(b)

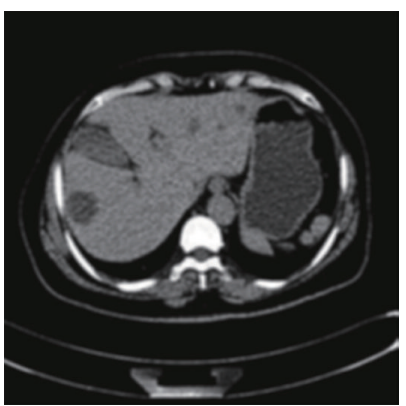

(e)

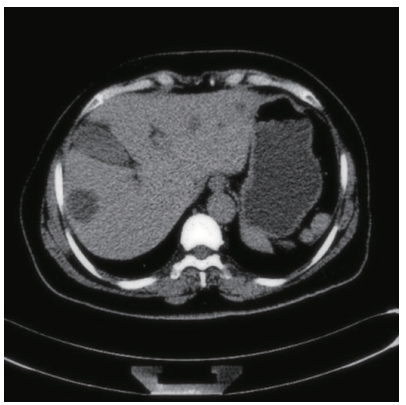

(h)

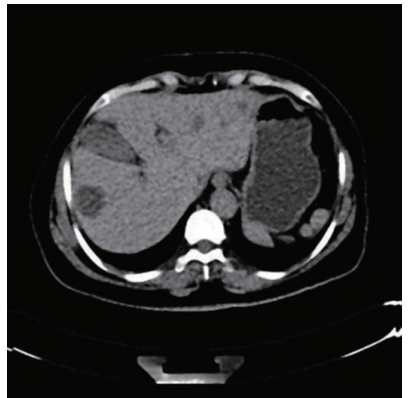

(c)

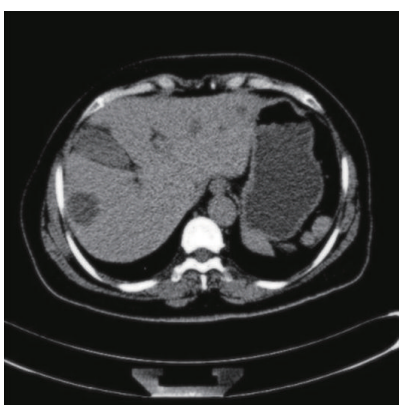

(f)

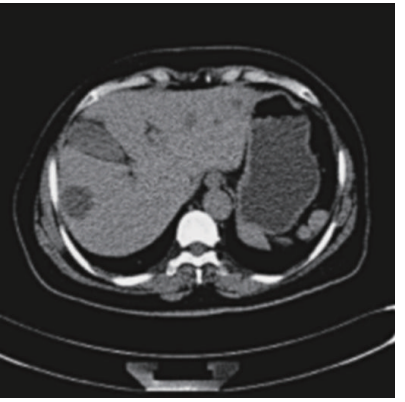

(i)

Figure 2: Abdominal CT images of a 62-year-old woman. (a) Original SDCT image with tube current time product $150 \mathrm{mAs}$. (b) Original LDCT image with tube current time product $60 \mathrm{mAs}$. (c) LDCT image processed by $5 \times 5$ median filter. (d) LDCT image processed by $5 \times 5$ wiener filter. (e) LDCT image processed by Gaussian smoothing with $[\sigma=1.8, \mu=0]$. (f) LDCT image processed by PMD with $[\sigma=2]$. (g) LDCT image processed by FPMD with $[\sigma=2, \alpha=0.2]$. (h) LDCT image processed by FPMD with $[\sigma=2, \alpha=0.5]$. (i) LDCT image processed by FPMD with $[\sigma=2, \alpha=1.5]$.

proposed FPMD. We can observe that, the noise grains and artifacts were significantly reduced for the FPMD processed L-CT images with suitable $\alpha$ both in Figures 1 and 2 . The fine anatomical/pathological features can be well preserved compared to the original SDCT images (Figures 1(a) and 2(a)) under standard dose conditions.

\section{Conclusions}

In this paper, we propose a new fractional-order PMD (FPMD) for L-CT sinogram imaging based on G-L fractional-order derivative definition. Since FPMD is a interpolation between 
integer-order Nonlinear Anisotropic Diffusion (NAD) and fourth-order partial differential equations, it provides a more flexible way to balance the noise reducing and anatomical details preserving. Smoothing results for phantoms and real sinograms show that FPMD with suitable parameters can suppress the staircase effects and speckle effects efficiently. In addition, FPMD also has good performance in visual quality and root mean square errors (RMSE).

\section{Acknowledgments}

This paper is supported by the National Natural Science Foundation of China (no. 60873102), Major State Basic Research Development Program (no. 2010CB732501), and Open Foundation of Visual Computing and Virtual Reality Key Laboratory Of Sichuan Province (no. J2010N03). This paper was supported by a Grant from the National High Technology Research and Development Program of China (no. 2009AA12Z140) and Open foundation of Key Laboratory of Land Resources Evaluation and Monitoring of Southwest Sichuan Normal University, Ministry of Education.

\section{References}

[1] D. J. Brenner and E. J. Hall, “Computed tomography-an increasing source of radiation exposure," New England Journal of Medicine, vol. 357, no. 22, pp. 2277-2284, 2007.

[2] J. Hansen and A. G. Jurik, "Survival and radiation risk in patients obtaining more than six CT examinations during one year," Acta Oncologica, vol. 48, no. 2, pp. 302-307, 2009.

[3] H. J. Brisse, J. Brenot, N. Pierrat et al., "The relevance of image quality indices for dose optimization in abdominal multi-detector row CT in children: experimental assessment with pediatric phantoms," Physics in Medicine and Biology, vol. 54, no. 7, pp. 1871-1892, 2009.

[4] L. Yu, "Radiation dose reduction in computed tomography: techniques and future perspective," Imaging in Medicine, vol. 1, no. 1, pp. 65-84, 2009.

[5] J. Weidemann, G. Stamm, M. Galanski, and M. Keberle, "Comparison of the image quality of various fixed and dose modulated protocols for soft tissue neck CT on a GE Lightspeed scanner," European Journal of Radiology, vol. 69, no. 3, pp. 473-477, 2009.

[6] W. Qi, J. Li, and X. Du, "Method for automatic tube current selection for obtaining a consistent image quality and dose optimization in a cardiac multidetector CT," Korean Journal of Radiology, vol. 10, no. 6, pp. 568-574, 2009.

[7] A. Kuettner, B. Gehann, J. Spolnik et al., "Strategies for dose-optimized imaging in pediatric cardiac dual source CT," Rofo, vol. 181, no. 4, pp. 339-348, 2009.

[8] P. Kropil, R. S. Lanzman, C. Walther et al., "Dose reduction and image quality in MDCT of the upper abdomen: potential of an adaptive post-processing filter," Rofo, vol. 182, no. 3, pp. 248-253, 2009.

[9] M. K. Kalra, M. M. Maher, M. A. Blake et al., "Detection and characterization of lesions on lowradiation-dose abdominal CT images postprocessed with noise reduction filters," Radiology, vol. 232, no. 3, pp. 791-797, 2004.

[10] H. B. Lu, X. Li, L. H. Li et al., "Adaptive noise reduction toward low-dose computed tomography," in Medical Imaging: Physics of Medical Imaging, vol. 5030 of Proceedings of the SPIE, pp. 759-766, 2003.

[11] M. K. Kalra, C. Wittram, M. M. Maher et al., "Can noise reduction filters improve low-radiation-dose chest CT images? Pilot study," Radiology, vol. 228, no. 1, pp. 257-264, 2003.

[12] M. K. Kalra, M. M. Maher, D. V. Sahani et al., "Low-dose CT of the abdomen: evaluation of image improvement with use of noise reduction filters-Pilot study," Radiology, vol. 228, no. 1, pp. 251-256, 2003.

[13] J. C. Ramirez Giraldo, Z. S. Kelm, L. S. Guimaraes et al., "Comparative study of two image space noise reduction methods for computed tomography: bilateral filter and nonlocal means," in Proceedings of the 31st Annual International Conference of the IEEE Engineering in Medicine and Biology Society: Engineering the Future of Biomedicine (EMBC '09), pp. 3529-3532, September 2009. 
[14] A. Manduca, L. Yu, J. D. Trzasko et al., "Projection space denoising with bilateral filtering and CT noise modeling for dose reduction in CT," Medical Physics, vol. 36, no. 11, pp. 4911-4919, 2009.

[15] N. Mail, D. J. Moseley, J. H. Siewerdsen, and D. A. Jaffray, "The influence of bowtie filtration on cone-beam CT image quality," Medical Physics, vol. 36, no. 1, pp. 22-32, 2009.

[16] M. Kachelrieß, O. Watzke, and W. A. Kalender, "Generalized multi-dimensional adaptive filtering for conventional and spiral single-slice, multi-slice, and cone-beam CT," Medical Physics, vol. 28, no. 4, pp. 475-490, 2001.

[17] G. F. Rust, V. Aurich, and M. Reiser, "Noise/dose reduction and image improvements in screening virtual colonoscopy with tube currents of $20 \mathrm{mAs}$ with nonlinear Gaussian filter chains," in Medical Imaging: Physiology and Function from Multidimensional Images, vol. 4683 of Proceedings of the SPIE, pp. 186-197, San Diego, Calif, USA, February 2002.

[18] Z. Liao, S. Hu, and W. Chen, "Determining neighborhoods of image pixels automatically for adaptive image denoising using nonlinear time series analysis," Mathematical Problems in Engineering, vol. 2010, Article ID 914564, 14 pages, 2010.

[19] H.-C. Hsin, T.-Y. Sung, and Y.-S. Shieh, "An adaptive coding pass scanning algorithm for optimal rate control in biomedical images," Computational and Mathematical Methods in Medicine, vol. 2012, Article ID 935914, 7 pages, 2012.

[20] H. Lu, I. T. Hsiao, X. Li, and Z. Liang, "Noise properties of low-dose CT projections and noise treatment by scale transformations," in Proceedings of the IEEE Nuclear Science Symposium Conference Record, vol. 1-4, pp. 1662-1666, November 2001.

[21] J. Xu and B. M. W. Tsui, "Electronic noise modeling in statistical iterative reconstruction," IEEE Transactions on Image Processing, vol. 18, no. 6, pp. 1228-1238, 2009.

[22] I. A. Elbakri and J. A. Fessler, "Statistical image reconstruction for polyenergetic X-ray computed tomography," IEEE Transactions on Medical Imaging, vol. 21, no. 2, pp. 89-99, 2002.

[23] P. J. La Rivière and D. M. Billmire, "Reduction of noise-induced streak artifacts in X-ray computed tomography through spline-based penalized-likelihood sinogram smoothing," IEEE Transactions on Medical Imaging, vol. 24, no. 1, pp. 105-111, 2005.

[24] P. J. La Rivière, "Penalized-likelihood sinogram smoothing for low-dose CT," Medical Physics, vol. 32, no. 6, pp. 1676-1683, 2005.

[25] J. Wang, H. Lu, J. Wen, and Z. Liang, "Multiscale penalized weighted least-squares sinogram restoration for low-dose X-ray computed tomography," IEEE Transactions on Biomedical Engineering, vol. 55, no. 3, pp. 1022-1031, 2008.

[26] P. Forthmann, T. Köhler, P. G. C. Begemann, and M. Defrise, "Penalized maximum-likelihood sinogram restoration for dual focal spot computed tomography," Physics in Medicine and Biology, vol. 52, no. 15, pp. 4513-4523, 2007.

[27] J. Wang, T. Li, H. Lu, and Z. Liang, "Penalized weighted least-squares approach to sinogram noise reduction and image reconstruction for low-dose X-ray computed tomography," IEEE Transactions on Medical Imaging, vol. 25, no. 10, pp. 1272-1283, 2006.

[28] Z. Liao, S. Hu, M. Li, and W. Chen, "Noise estimation for single- slice sinogram of low-dose X-ray computed tomography using homogenous patch," Mathematical Problems in Engineering, vol. 2012, Article ID 696212, 16 pages, 2012.

[29] H. B. Lu, X. Li, I. T. Hsiao, and Z. G. Liang, "Analytical noise treatment for low-dose CT projection data by penalized weighted least-square smoothing in the K-L domain," in Medical Imaging: Physics of Medical Imaging, vol. 4682 of Proceedings of the SPIE, pp. 146-152, 2002.

[30] P. Perona and J. Malik, "Scale-space and edge detection using anisotropic diffusion," IEEE Transactions on Pattern Analysis and Machine Intelligence, vol. 12, no. 7, pp. 629-639, 1990.

[31] A. M. Mendrik, E. J. Vonken, A. Rutten, M. A. Viergever, and B. Van Ginneken, "Noise reduction in computed tomography scans using 3-D anisotropic hybrid diffusion with continuous switch," IEEE Transactions on Medical Imaging, vol. 28, no. 10, pp. 1585-1594, 2009.

[32] T. S. Kim, C. Huang, J. W. Jeong, D. C. Shin, M. Singh, and V. Z. Marmarelis, "Sinogram enhancement for high-resolution ultrasonic transmission tomography using nonlinear anisotropic coherence diffusion," in Proceedings of the IEEE Ultrasonics Symposium, vol. 1, pp. 1816-1819, October 2003.

[33] M. Ceccarelli, V. De Simone, and A. Murli, "Well-posed anisotropic diffusion for image denoising," IEE Proceedings, vol. 149, no. 4, pp. 244-252, 2002.

[34] V. B. Surya Prasath and A. Singh, "Well-posed inhomogeneous nonlinear diffusion scheme for digital image denoising," Journal of Applied Mathematics, vol. 2010, Article ID 763847, 14 pages, 2010. 
[35] Z. Liao, S. Hu, D. Sun, and W. Chen, "Enclosed laplacian operator of nonlinear anisotropic diffusion to preserve singularities and delete isolated points in image smoothing," Mathematical Problems in Engineering, vol. 2011, Article ID 749456, 15 pages, 2011.

[36] T. Li, X. Li, J. Wang et al., "Nonlinear sinogram smoothing for low-dose X-ray CT," IEEE Transactions on Nuclear Science, vol. 51, no. 5, pp. 2505-2513, 2004.

[37] S. Hu, Zh. Liao, D. Sun, and W. Chen, "A numerical method for preserving curve edges in nonlinear anisotropic smoothing," Mathematical Problems in Engineering, vol. 2011, Article ID 186507, 14 pages, 2011.

[38] Y. L. You and M. Kaveh, "Fourth-order partial differential equations for noise removal," IEEE Transactions on Image Processing, vol. 9, no. 10, pp. 1723-1730, 2000.

[39] Y. Zhang, H. D. Cheng, Y. Q. Chen, and J. Huang, "A novel noise removal method based on fractional anisotropic diffusion and subpixel approach," New Mathematics and Natural Computation, vol. 7, no. 1, pp. 173-185, 2011.

[40] D. Chen, Y. Q. Chen, and D. Xue, “Digital fractional order Savitzky-Golay differentiator and its application," in Proceedings of the ASME International Design Engineering Technical Conferences E Computers and Information in Engineering Conference (IDETC/CIE '11), Washington, DC, USA, August 2011.

[41] L.-T. Ko, J.-E. Chen, Y.-S. Shieh, M. Scalia, and T.-Y. Sung, "A novel fractional discrete cosine transform based reversible watermarking for healthcare information management systems," Mathematical Problems in Engineering, vol. 2012, Article ID 757018, 2012.

[42] T. F. Chan, S. Esedoglu, and F. Park, "A fourth order dual method for staircase reduction in texture extraction and image restoration problems," in Proceedings of the 17th IEEE International Conference on Image Processing (ICIP '10), pp. 4137-4140, September 2010.

[43] T. Chan, A. Marquina, and P. Mulet, "High-order total variation-based image restoration," SIAM Journal on Scientific Computing, vol. 22, no. 2, pp. 503-516, 2001.

[44] Z. Jun and W. Zhihui, "A class of fractional-order multi-scale variational models and alternating projection algorithm for image denoising," Applied Mathematical Modelling, vol. 35, no. 5, pp. 2516-2528, 2011.

[45] M. Li and W. Zhao, "Visiting power laws in cyber-physical networking systems," Mathematical Problems in Engineering, vol. 2012, Article ID 302786, 13 pages, 2012.

[46] M. Li, C. Cattani, and S. Y. Chen, "Viewing sea level by a one-dimensional random function with long memory," Mathematical Problems in Engineering, vol. 2011, Article ID 654284, 2011.

[47] M. Li, "Fractal time series-a tutorial review," Mathematical Problems in Engineering, vol. 2010, Article ID 157264, 2010.

[48] Y. F. Pu, "Application of fractional differential approach to digital image processing," Journal of Sichuan University, vol. 39, no. 3, pp. 124-132, 2007.

[49] J. Bai and X. C. Feng, "Fractional-order anisotropic diffusion for image denoising," IEEE Transactions on Image Processing, vol. 16, no. 10, pp. 2492-2502, 2007.

[50] P. Perona and J. Malik, "Scale-space and edge detection using anisotropic diffusion," IEEE Transactions on Pattern Analysis and Machine Intelligence, vol. 12, no. 7, pp. 629-639, 1990.

[51] Y. Chen, W. Chen, X. Yin et al., "Improving low-dose abdominal CT images by Weighted Intensity Averaging over Large-scale Neighborhoods," European Journal of Radiology, vol. 80, no. 2, pp. e42-e49, 2011. 


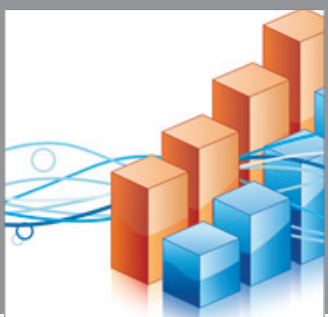

Advances in

Operations Research

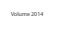

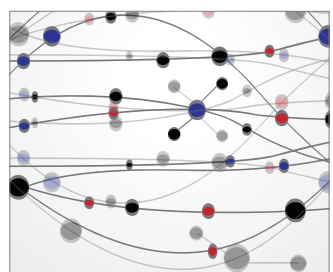

\section{The Scientific} World Journal
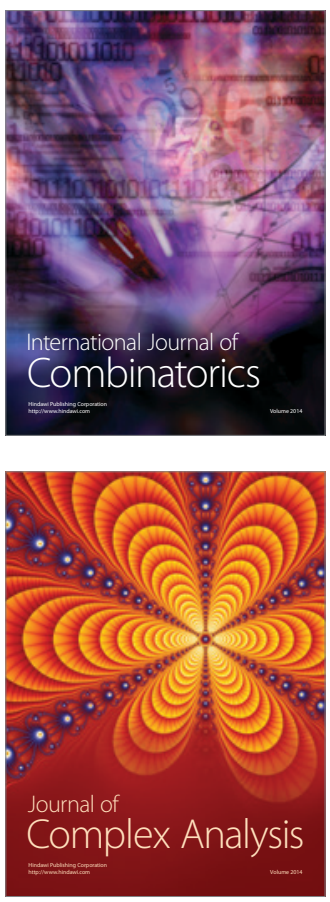

International Journal of

Mathematics and

Mathematical

Sciences
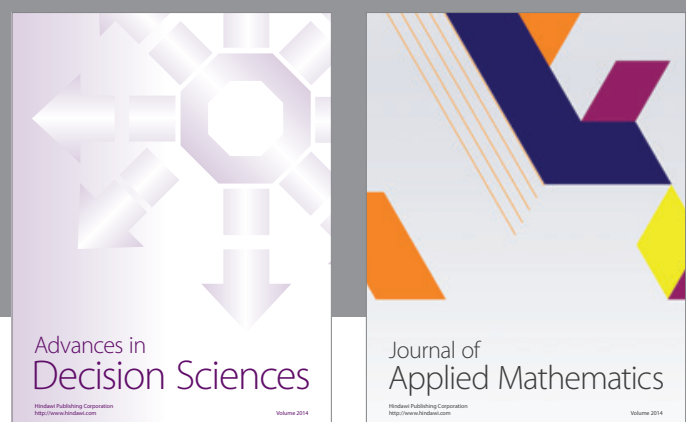

Journal of

Applied Mathematics
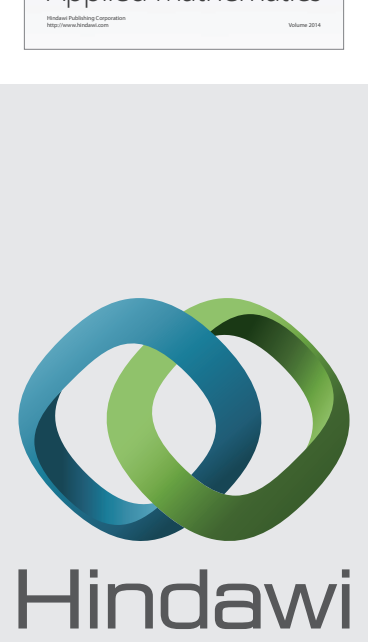

Submit your manuscripts at http://www.hindawi.com
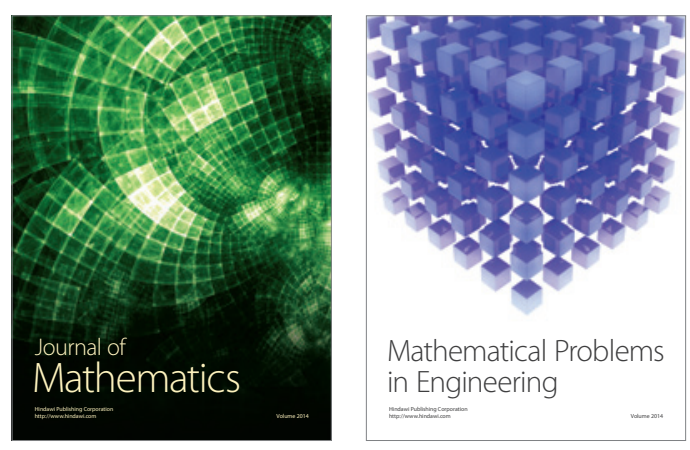

Mathematical Problems in Engineering
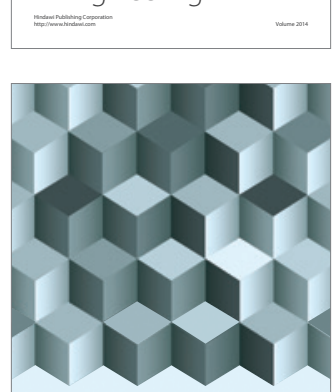

Journal of

Function Spaces
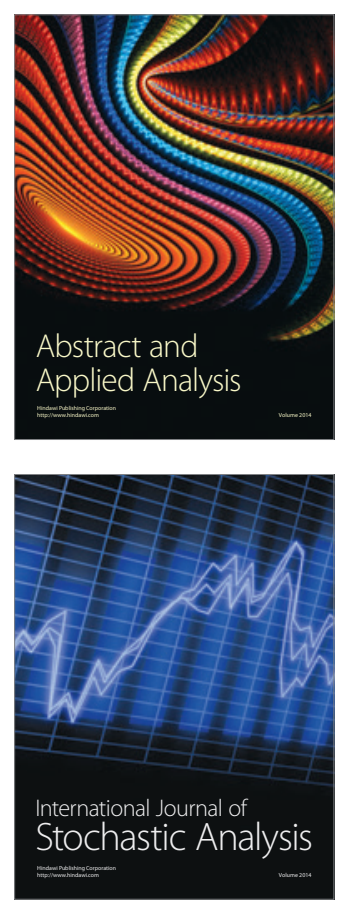

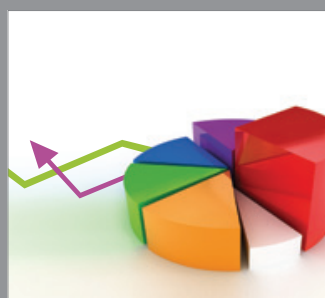

ournal of

Probability and Statistics

Promensencen
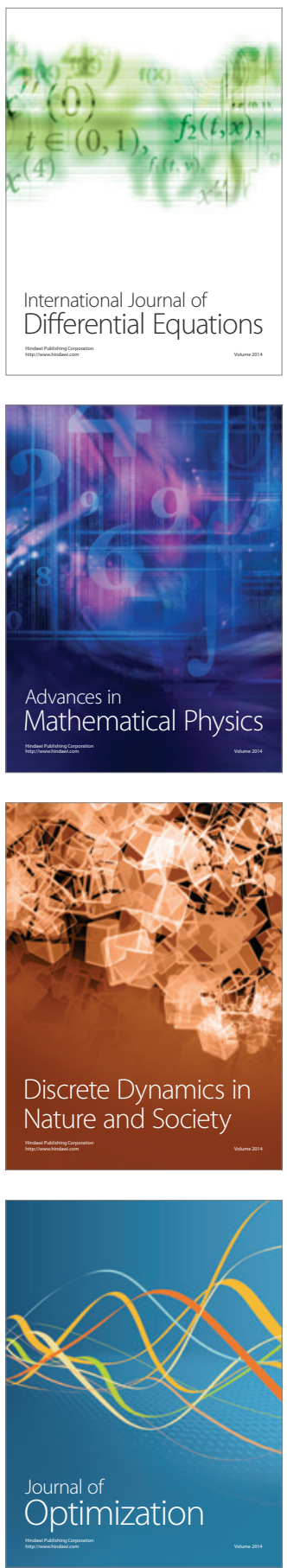